\title{
Different therapeutic effects of distinct KISS1 fragments on breast cancer in vitro and in vivo
}

\author{
GUO-QING SONG and YI ZHAO \\ Department of Pancreatic and Breast Surgery, Shengjing Hospital of China Medical University, \\ Shenyang, Liaoning 110001, P.R. China
}

Received May 24, 2013; Accepted July 7, 2013

DOI: 10.3892/ijo.2013.2029

\begin{abstract}
Breast cancer is the most frequently diagnosed cancer in women. In these studies, a metastasis suppressor gene, KISS1 and its truncated fragment, were overexpressed in the breast cancer cell line MDA-MB-231. In addition, KISS1 expression was downregulated in MDA-MB-157 cell line using a KISS1-specific siRNA. The effects of KISS1 on breast cancer cells both in vivo and in vitro were then identified. Our results indicate that KISS1 can induce apoptosis and inhibit mobility of breast cancer cells. Moreover, the expression of KISS1 in established xenografted tumors was associated with a decrease in tumor size and weight. Accordingly, the survival rate of these mice was significantly higher compared to that of mice bearing tumors that did not express KISS1. We also confirm that KISS1 could decrease the number of circulating tumor cells (CTCs). The plasma levels of metastin and the number of CTCs were significantly positively correlated. Furthermore, we found that KISS1 can inactivate p-MEK and p-ERK. Overall, these studies demonstrate the antitumor activity of KISS1 in the breast cancer cell lines and provide insight into relevant mechanisms that may lead to novel treatments for breast cancer.
\end{abstract}

\section{Introduction}

Cancer is the leading cause of death in economically developed countries and the second leading cause of death in developing countries (1). Breast cancer is the most frequently diagnosed cancer in females. Worldwide, more than a million women are diagnosed with breast cancer in 2008, accounting for $14 \%$ $(458,400)$ of the total cancer deaths (2). Maintaining a healthy body weight, increasing physical activity and minimizing

Correspondence to: Dr Yi Zhao, Department of Pancreatic and Breast Surgery, Shengjing Hospital of China Medical University, 36 Sanhao Street, Heping District, Shenyang, Liaoning 110004, P.R. China

E-mail: zhaozhaoyi_sj@163.com

Key words: KISS1, breast cancer, survival analysis, circulating tumor cells, mobility alcohol intake are the best available strategies to reduce the risk of developing breast cancer (3).

KISSI was originally identified as a metastasis suppressor gene in human melanoma and breast carcinoma cells (4). The KISS1 gene is located on chromosome 1 near q32.1 with regulatory elements localized in chromosome 6 at 6q16.3-q23 (5). The KISS1 gene encodes a precursor protein that is processed into several related peptides, generically named kisspeptins $(6,7)$, where the major product appears to be a 54-amino acid peptide, named kisspeptin-54 or metastin. In addition, three natural peptides of 14-, 11- and 10-amino acids have been also identified, sharing a common 10 -amino acid C-terminal region (8). Cogent data support that loss of KISS1 expression has been associated with the progression and metastasis of various tumors, including esophageal, brain, breast, ovarian and melanoma (9). KISSI was recently shown epigenetically silenced by hypermethylation in bladder cancer (10) and colorectal cancer (11).

Circulating tumor cells (CTCs) captured from peripheral blood were recently shown to predict disease outcome and therapy response in cancer patients (12). The enumeration of CTCs at different time-points during treatment has proven to be a reliable surrogate marker of treatment response and a potential alternative for non-invasive therapy monitoring (13). EpCAM as well as cytokeratin expressing cells can be found in peripheral blood of advanced cancer patients but are rare in healthy donors (14). Breast cancer cells of all grades typically express the epithelial cytokeratins CK7, CK8, CK18 and CK19 (15).

In this report, we evaluated whether KISS-1 expression and methylation patterns differed in breast cancer cell lines, MDA-MB-231 and MDA-MB-157. Then we evaluated the inhibition potential of KISS1 protein on tumor growth in a breast cancer model, as evidenced by tumor size, tumor weight and circulating tumor cells. Furthermore, mechanism(s) of an integrated KISS1 protein, as well as one truncated KISS1 protein, were examined in a breast cancer model. It is anticipated that the results obtained will provide novel insight into potential strategies for the treatment of breast cancer.

\section{Materials and methods}

Cell culture. MDA-MB-231 and MDA-MB-157 cell lines were purchased from the American Type Culture Collection 
(Rockville, MD, USA) and maintained in DMEM supplemented with $10 \%$ FCS and antibiotics $(100 \mu \mathrm{M}$ penicillin and $100 \mu \mathrm{M}$ streptomycin). Cells were maintained in a humidified cell incubator with $5 \% \mathrm{CO}_{2}$ at $37^{\circ} \mathrm{C}$.

DNA extraction, sodium bisulfite conversion and methylationspecific PCR (MSP). Cells were incubated with medium containing $10 \mu \mathrm{M}$ 5-aza-2'-deoxycytidine (5-aza-dC) (SigmaAldrich, Carlsbad, CA, USA) for $48 \mathrm{~h}$. Then we isolated DNA and carried out MSP as the method described below. Genomic DNA was extracted from cells using a TissueGen DNA kit according to the manufacturer's instructions (CWbiotech, Beijing, China). The concentration and purity of the DNA were determined by absorbance at 260 and $280 \mathrm{~nm}$ by NanoDrop $^{\text {TM }} 1000$ Spectrophotometer (Thermo Scientific, Wilmington, NC, USA). Sodium bisulfite modification for the extracted DNA was performed using an EZ DNA Methylation ${ }^{\mathrm{TM}}$ kit strictly according to the manufacturer's instructions (Zymo Research, Orange, CA, USA). Sequencing results confirmed that $>99.0 \%$ of cytosine residues were converted. The bisulfiteconverted DNA was resuspended in $10 \mu \mathrm{l}$ elution buffer and stored at $-80^{\circ} \mathrm{C}$ until the samples were ready for analysis. The $5 \mu 1$ PCR mixture contained $10 \mathrm{ng}$ bisulfite-treated DNA, $25 \mathrm{mM}$ dNTP, $0.2 \mathrm{U}$ of Hot Start Taq DNA polymerase (Takara, Dalian, China) and a $1 \mu \mathrm{M}$ mixture of forward and reverse primers. The primer sequences for the methylated KISS1 gene were 5'-CGGGTTGGAAGTTTTAGC-3' (sense) and 5'-GCTTCGACAAACGAAAAAC-3' (antisense) and for the unmethylated allele were 5'-TTTTGGGTTGGAAGT TTTAGT-3' (sense) and 5'-ACTTCAACAAACAAAAAAC AAC-3' (antisense). The PCR products were separated in $2 \%$ agarose gel with ethidium bromide and visualized under a UV imaging system (UVP, Upland, CA, USA).

RNA isolation and reverse transcriptase-polymerase chain reaction $(R T-P C R)$. RNA $(1 \mu \mathrm{g})$ was reverse-transcribed using AMV reverse transcriptase (Promega, Madison, WI, USA) and amplified using specific primers and conditions for KISS1. The KISS1 primers used were: 5'-ATGAACTCACT GGTTTCTTGGCAG-3' (sense) and 5'-TCACTGCCCCGCAC CTG-3' (antisense). GAPDH was used as an internal normalization control. The GAPDH primers used were: 5'-GAAGGCT GGGGCTCATTT-3' (sense) and 5'-GGGGCCATCCACA GTCTT-3' (antisense). PCR amplification of cDNA was performed in reaction volumes of $15 \mu \mathrm{l}$. Finally, products were resolved by $1 \%$ agarose gel electrophoresis and visualized by ethidium bromide staining and a UV imaging system (UVP).

siRNA and plasmid transfections. MDA-MB-231 cells were seeded in $10-\mathrm{cm}$ dishes and grown overnight to $70 \%$ confluency, trypsinized and transfected with siRNA targeting KISS1, or a non-targeting construct, using Amaxa nucleofector (Lonza, Portsmouth, NH, USA). For the preparation of truncated KISS1 proteins, the relevant sequences were amplified from full-length KISS1 by PCR using primers that included designed restriction sites (Table I), then digested with the relevant restriction enzymes and ligated into pcDNA3.1. MDA-MB-231 cells were seeded overnight and transfected with either pcDNA3.1 or pcDNA-3.1-KISS1 fragments, using
Lipofectamine $^{\mathrm{TM}} 2000$ according to the procedures of the manufacturer (Invitrogen, Carlsbad, CA, USA).

Immunofluorescence assays. Cells were washed with PBS, fixed with $4 \%$ paraformaldehyde, permeabilized in $2 \%$ Triton X-100 for 10 min, then blocked with 5\% bovine serum albumin in PBS containing 2\% Triton X-100 for $1 \mathrm{~h}$. AntiKISS1 antibodies (Santa Cruz Biotechnology, Santa Cruz, CA, USA) were incubated with cells for $2 \mathrm{~h}$ at room temperature to identify the location of intact KISS1 proteins, or truncated KISS1 fragments. Cells were then washed with PBS and incubated with Alexa Fluor ${ }^{\circledR} 488$ goat anti-rabbit $\operatorname{IgG}$ for $2 \mathrm{~h}$ at RT. To determine whether or not a lamellipodium was present, cells were stained for actin and actin-associated proteins using Fluorophore-conjugated phalloidin (Invitrogen). Cells were examined and photographed using a laser confocal microscope (Olympus, Tokyo, Japan).

Apoptosis assay. For the apoptosis assay, equal numbers of cells were seeded in 6-cm plates. Following the manufacturer's instructions (Apoptosis Detection kit, KeyGen, Nanjing, China), cells were trypsinized, washed twice with cold PBS, then resuspended in $200 \mu \mathrm{l}$ binding buffer. Annexin V-FITC was added to a final concentration of $0.5 \mu \mathrm{g} / \mathrm{ml}$. Additionally samples were incubated at room temperature in the dark. After $20 \mathrm{~min}, 300 \mu \mathrm{l}$ binding buffer containing $0.5 \mu \mathrm{g} / \mathrm{ml}$ PI was added and samples were immediately analyzed on a FACSCalibur flow cytometer (Becton-Dickinson Medical Devices, Shanghai, China). Cells in the stages of early apoptosis were defined as $\mathrm{FITC}^{+} / \mathrm{PI}^{-}$cells.

Gelatin zymography. Fifty micrograms of protein was applied to $10 \%$ polyacrylamide gels with $1 \%$ gelatin incorporated as a substrate for gelatinolytic proteases. After running the gel the SDS was removed by washing twice in $2.5 \%$ Triton X-100 for $30 \mathrm{~min}$. The gels were incubated overnight in zymography development buffer containing $50 \mathrm{mM}$ Tris- $\mathrm{HCl}(\mathrm{pH} 7.4)$, $2 \mathrm{mM} \mathrm{NaN}_{3}$ and $5 \mathrm{mM} \mathrm{CaCl}$. After development the gels were stained for $3 \mathrm{~h}$ in $45 \%$ methanol $/ 10 \%$ glacial acetic acid containing $1 \%(\mathrm{w} / \mathrm{v})$. Coomassie Blue R-250 and subsequently partially destained with the same solution without dye. The gelatinolytic activity of each MMP was qualitatively evaluated as a clear band against the blue stained gelatin background.

Xenograft assays. NOD SCID mice (NOD.CB17-Prkdcscid/ $\mathrm{NcrCrl}$ ) that were 3-5-weeks-old were purchased from Charles River (MA, USA). All animals were housed four to a plastic cage with filter top. The animal room was controlled at $20 \pm 2^{\circ} \mathrm{C}$, $50 \pm 10 \%$ humidity and a 12-h light/dark cycle. MDA-MB-231 cells $\left(4 \times 10^{7}\right.$ in $\left.200 \mu \mathrm{l}\right)$ or MDA-MB-157 cells $\left(5 \times 10^{7}\right.$ in $\left.200 \mu \mathrm{l}\right)$ were subcutaneously injected into the axilla of each mouse. After the tumor diameters reached 3-5 mm, the mice injected with MDA-MB-157 cells were divided randomly into three groups and received a $200 \mu \mathrm{l}$ intratumoral injection of siRNA, or scramble. The mice injected with MDA-MB-231 cells were divided randomly into four groups and received a $200 \mu \mathrm{l}$ intratumoral injection of pCDNA-3.1, pCDNA-3.1-KISS1-T1 or pCDNA-3.1-KISS1-T2. Two injections were administered at 9 a.m. and 4 p.m. every three days. Tumor growth was then monitored for 30 days. Every five days until the end of 
Table I. Primers used to generate integrated and truncated forms of KISS1.

\begin{tabular}{ll} 
Region of KISS1 amplified & \multicolumn{1}{c}{ Primers (5'-3') } \\
\hline aa 1-121 (integrated) & F, AAGCTTATGAACTCACTGGTTTCTTGGC \\
& R, GGATTCCTTCGCGTCCGGCTTCCTCAAG \\
aa 20-121 (truncated) & F, AAGCTTGAGCCATTA GAAAAGGTG \\
& R, GGATTCCTTCGCGTCCGGCTTCCTCAAG
\end{tabular}

Product (bp)

Italic indicates HindIII restriction sites; underlined indicates BamHI restriction sites. F, forward; R, reverse.

the experiment, one mouse from each group was randomly selected to be sacrificed by $\mathrm{CO}_{2}$ asphyxiation and tumors were harvested and weighed. The tissue was fixed in $4 \%$ paraformaldehyde for histopathology analysis. All experiments with animals were performed according to the guidelines of China Medical University Ethics Committee.

Immunohistochemical staining (IHC). Immunohistochemical staining was performed on $4-\mu \mathrm{m}$ sections obtained from formalin-fixed, paraffin-embedded blocks. Endogenous peroxidase activity was blocked with $3 \%$ hydrogen peroxide for $30 \mathrm{~min}$. Antigen retrieval was carried out in citrate buffer (10 mM, pH 6.0) for $30 \mathrm{~min}$ at $95^{\circ} \mathrm{C}$ in a microwave oven. Sections were incubated with primary antibody at $4^{\circ} \mathrm{C}$ overnight. Signaling pathway proteins were probed with: anti-ERK, anti-phospho-ERK, anti-MEK and anti-phospho-MEK antibodies (Santa Cruz). Afterward, sections were incubated with a biotinylated secondary antibody and then exposed to a streptavidin complex (HRP). Positive reactions were visualized with 3, 3'-diaminobenzidine tetrahydrochloride (DAB, Sigma), followed by counterstaining with hematoxylin. Sections treated without primary antibodies were used as negative controls.

Survival curves. Additional mice $(n=140)$ were used to establish xenografts to obtain survival curves. Mice with xenografted tumors (as described above) that reached 3-5 mm in diameter were divided into seven groups ( $n=20$ for each). Survival was monitored until the experiments were terminated due to heavy tumor burden.

Isolation and enumeration of circulating tumor cells. The CellSearch ${ }^{\circledR}$ system (Veridex LLC, Warren, NJ, USA) is the only US Food and Drug Administration-cleared test for CTC enumeration in clinical practice (16). Blood samples $(7.5 \mathrm{ml})$ from breast mouse models were drawn into CellSave ${ }^{\circledR}$ tubes (Veridex LLC), which were maintained at RT and processed within $72 \mathrm{~h}$ of collection. CTCs were defined as nucleated EpCAM-positive cells, lacking CD45 but expressing cytoplasmic cytokeratins 8, 18 and 19. All CTC evaluations were performed by qualified and trained personnel.

Enzyme-linked immuno sorbent assay (ELISA). Blood samples for metastin determination were collected in ethylenediamine tetraacetate tubes, placed on ice and centrifuged at 3,000 rpm for $20 \mathrm{~min}$. Recovered serum was stored at $-80^{\circ} \mathrm{C}$ in aliquots until assayed. All samples were measured in duplicate. Circulating serum metastin was determined using a sandwich enzyme immunoassay (Human Metastin ELISA Phoenix Pharmaceuticals, Burlingame, CA, USA) with a sensitivity of $0.12 \mathrm{ng} / \mathrm{ml}$.

Statistical analysis. Data are presented as the mean \pm standard deviation (SD). Differences between groups were analyzed using Student's t-test for continuous variables. Statistical analysis was performed using Statistical Package for the Social Sciences (SPSS, version 17.0; SPSS, Inc.) and significance was established at $\mathrm{P}<0.05$.

\section{Results}

The $m R N A$ and protein levels of KISS1 were evaluated in $M D A-M B-231$ and MDA-MB-157 cells after treatment. We compared endogenous KISS1 protein level in two breast cancer cell lines, MDA-MB-231 and MDA-MB-157. MDA-MB-231 cells showed relatively low KISS1 expression levels, whereas the MDA-MB-157 lines expressed relatively stronger KISS1 protein levels (Fig. 1A). A further link between methylation and gene silencing was established by the treatment of hypermethylated (MDA-MB-231) and hypomethylated (MDA-MB-157) cell lines with a DNA demethylating drug. Exposure of the two cell lines to 5-aza-2'-deoxycytidine (AZA) increased the expression of KISS1 at the transcript level (Fig. 1B). Based on the level of KISS1 protein in MDA-MB-231 and MDA-MB-157 cells, MDA-MB-231 cells were transfected with KISS1 fragments that included: integrated (aa 1-121) and truncated (aa 20-121). MDA-MB-157 cells were transfected with KISS1 siRNA. After transfection, KISS1 mRNA and protein levels were significantly changed compared to untransfected cells by using RT-PCR and western blotting, respectively (Fig. 1A). These results collectively suggested that the transfection was successful. Interesting, the results of immunofluorescence analysis showed integrated KISS1 protein was localized in the cytoplasm of transfected MDA-MB-231 cells, however, truncated KISS1 protein was mainly localized in the nucleus (Fig. 1C).

The effects of KISS1 protein on breast cancer cells in vitro. To detect apoptotic cells, Annexin V-FITC and PI double staining was performed. In MDA-MB-231 cells expressing intact or truncated KISS1, the apoptotic ratio was 4-5 times higher than that of untransfected ones $(\mathrm{P}<0.05$; Fig. $2 \mathrm{~A})$. The effect of 
A
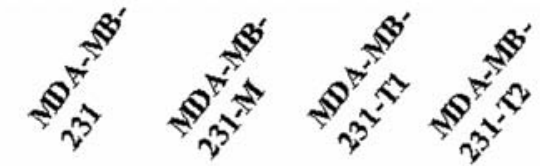

KISS1

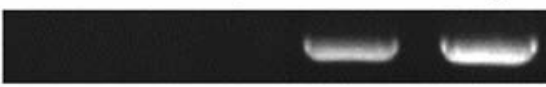

KISS1

GAPDH

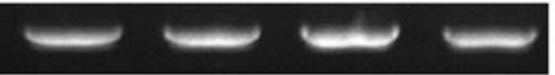

GAPDH
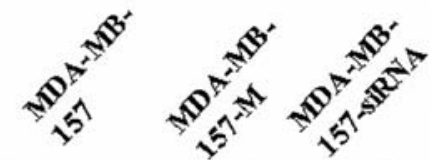

KISS1
cytoplasm

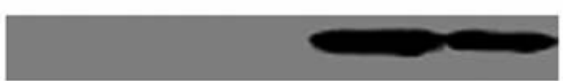

KISS1

nucleus

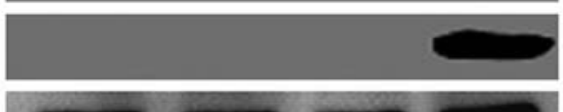

cytoplasm

$\beta$-actin

$\beta$-actin

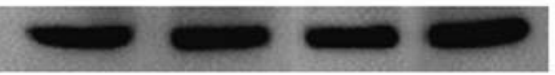

MMP-2

MMP-2

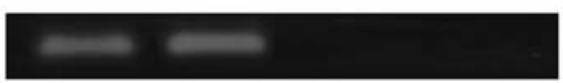

MMP-9

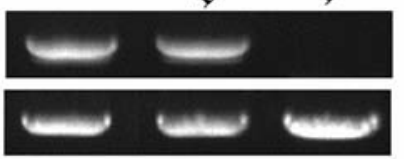

MMP-9
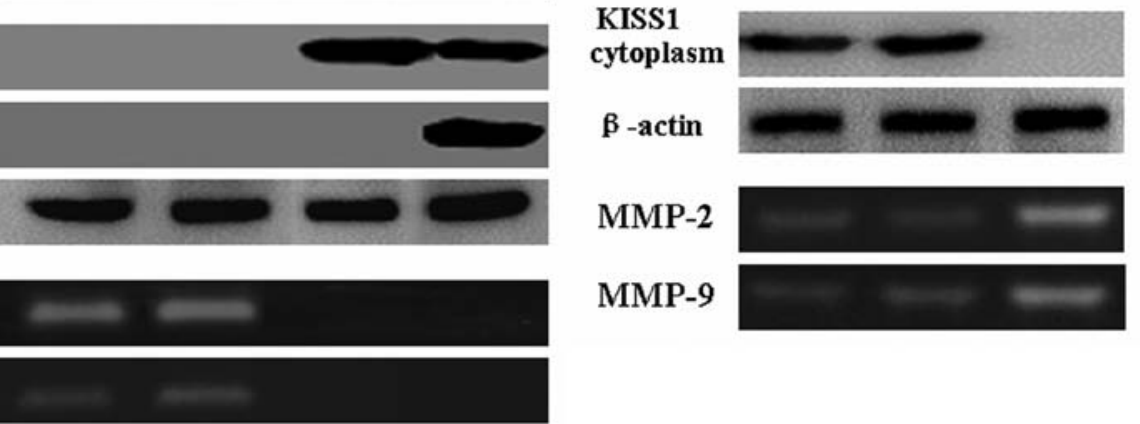

B
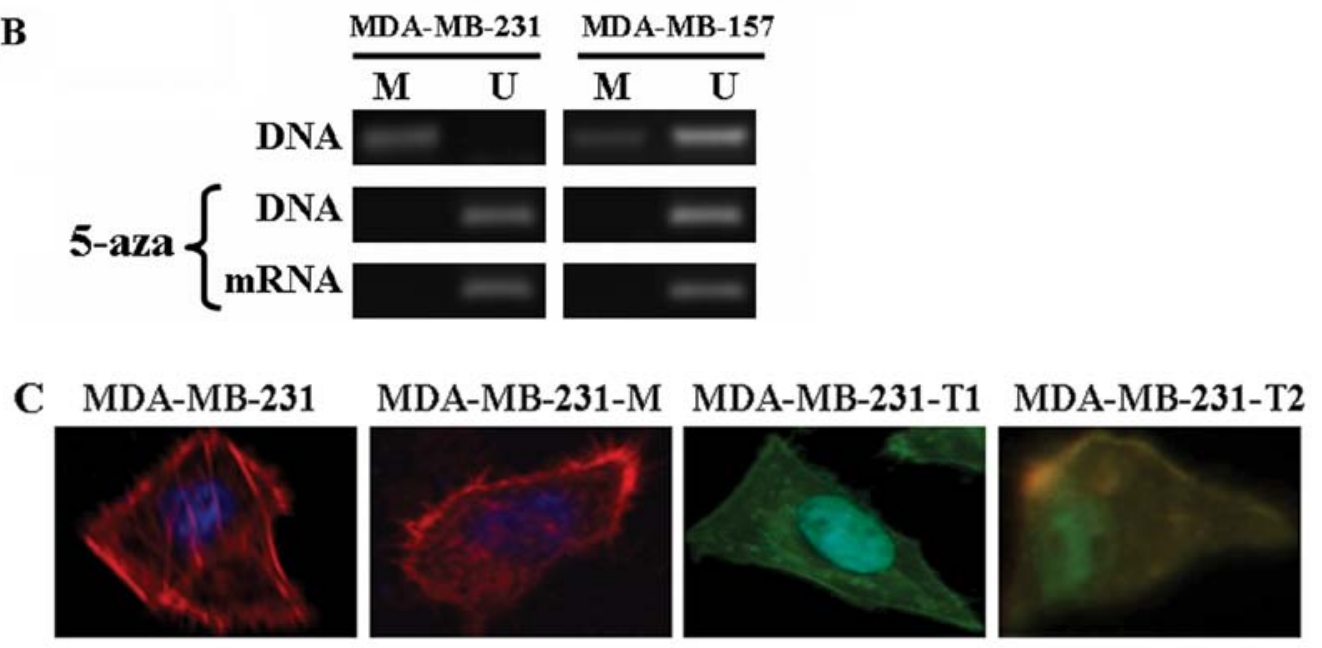

MDA-MB-157

MDA-MB-157-M

MDA-MB-157-siRNA
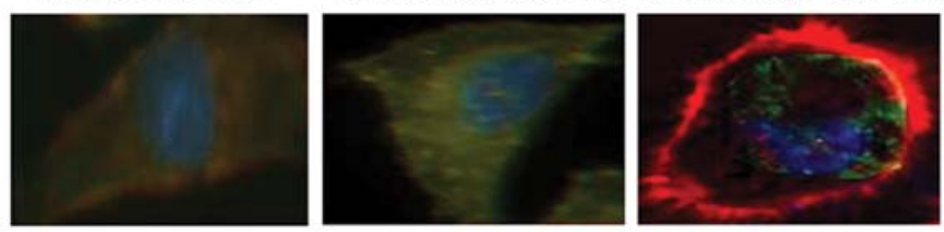

Figure 1. Detection of KISS1 following transfection. (A) KISS1 mRNA and protein levels in MDA-MB-231 and MDA-MB-157 cells and transfected ones detected using RT-PCR and western blot analysis, respectively. Gelatinolytic activity of secreted active of MMP-2 and MMP-9 of breast cancer cells was analyzed by zymography. (B) KISS1 promoter methylation in MDA-MB-231 and MDA-MB-157 cells was attributed to silenced or low expression of REIC expression. U, unmethylated; M, methylated; 5-aza, 5-aza-2'-deoxycytidine. (C) Detection of KISS1 in transfected and untransfected breast cancer cells by immunofluorescence. Intact KISS1 proteins were located predominantly in the cytoplasm. Truncated KISS1 fragments were observed to localize in the nucleus. Phalloidin was employed to observe the lamellipodia. Nuclei were stained with DAPI. M, mock transfection; T1, intact KISS1 protien; T2, KISS1 $20-121$.

KISS1-expression on the motility of MDA-MB-231 cells was determined by wound-healing assay and transwell assay. The percentage wound closure of the MDA-MB-231 cells transfected with intact $(89.4 \%)$ or truncated KISS1 $(88.7 \%)$ was decreased when compared to the untreated (66.4\%) and the mock transfected $(63.6 \%)$ cells $(\mathrm{P}<0.05$; Fig. 2B). Transwell assay also showed that less KISS1 positive cells migrated to the lower side of the membrane than negative ones $(\mathrm{P}<0.05$;
Fig. 2C). Furthermore, we found the activity of MMP-2 and MMP-9 was inhibited by both intact and truncated KISS1 proteins (Fig. 1A, blue stripe). Conversely, MDA-MB-157 cells treated with KISS1 siRNA showed less apoptotic ratio than untreated ones ( $\mathrm{P}<0.05$; Fig. 2A). Motility was significantly increased in KISS1 siRNA treated cells compared to KISS1 scramble treated or untreated cells $(\mathrm{P}<0.05$; Fig. 2B and C). Additionally, forced KISS1 overexpression could cause 
A MDA-MB-231

MDA-MB-231-M MDA-MB-231-T1 MDA-MB-231-T2
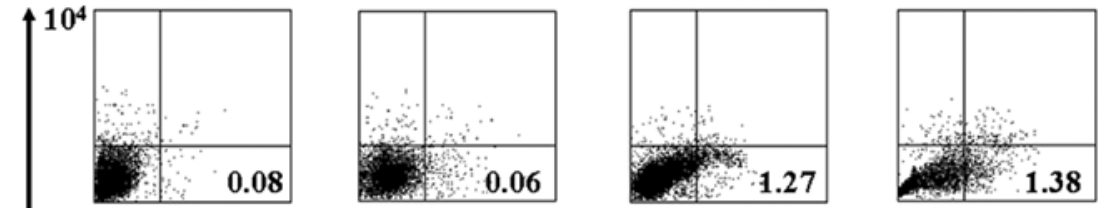

MDA-MB-157 MDA-MB-157-M MDA-MB-157-siRNA
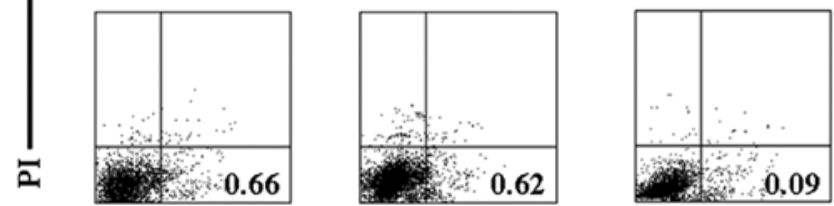

Annexin V-FITC

$10^{4}$

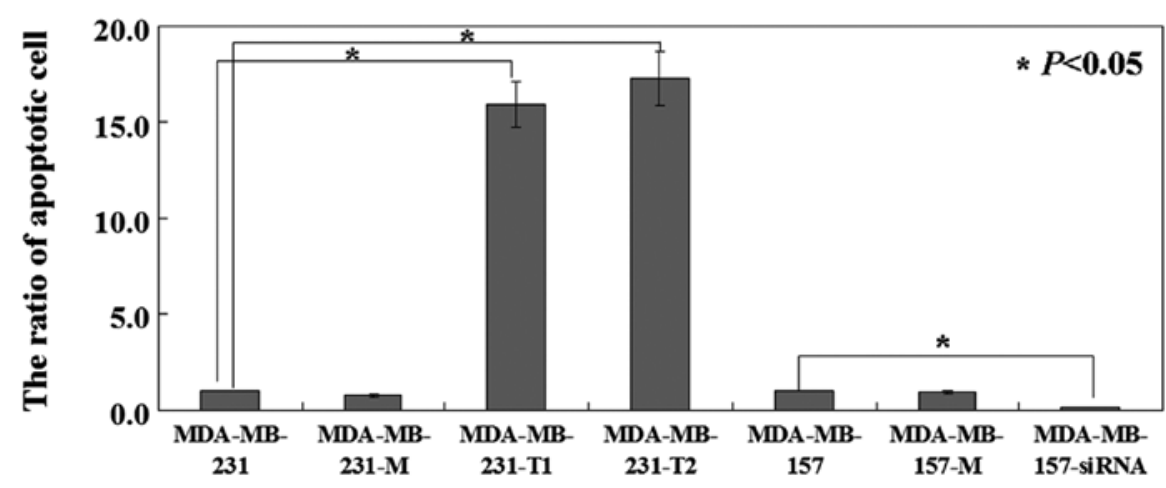

$\mathbf{B}$

MDA-MB-231 MDA-MB-231-M MDA-MB-231-T1 MDA-MB-231-T2

$\mathbf{0 ~ h}$
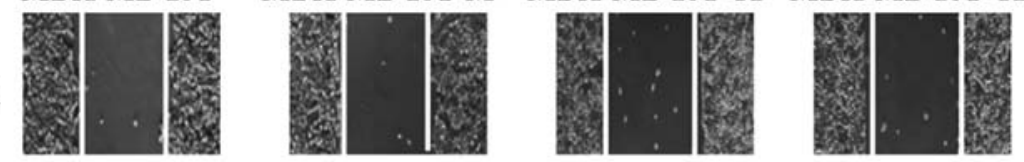

$24 \mathrm{~h}$
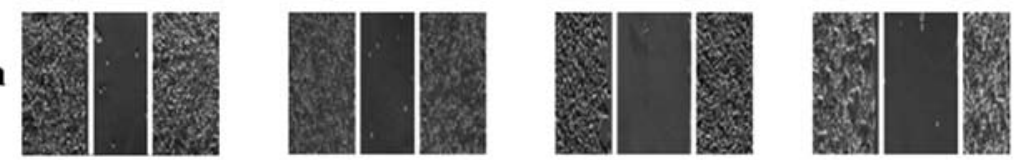

MDA-MB-157

MDA-MB-157-M MDA-MB-157-siRNA

$\mathbf{0 ~ h}$
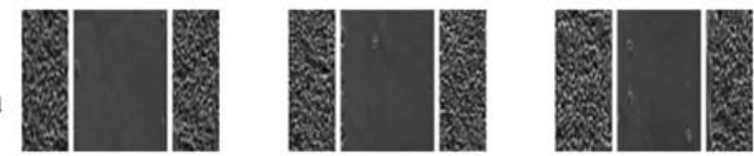

$24 \mathrm{~h}$
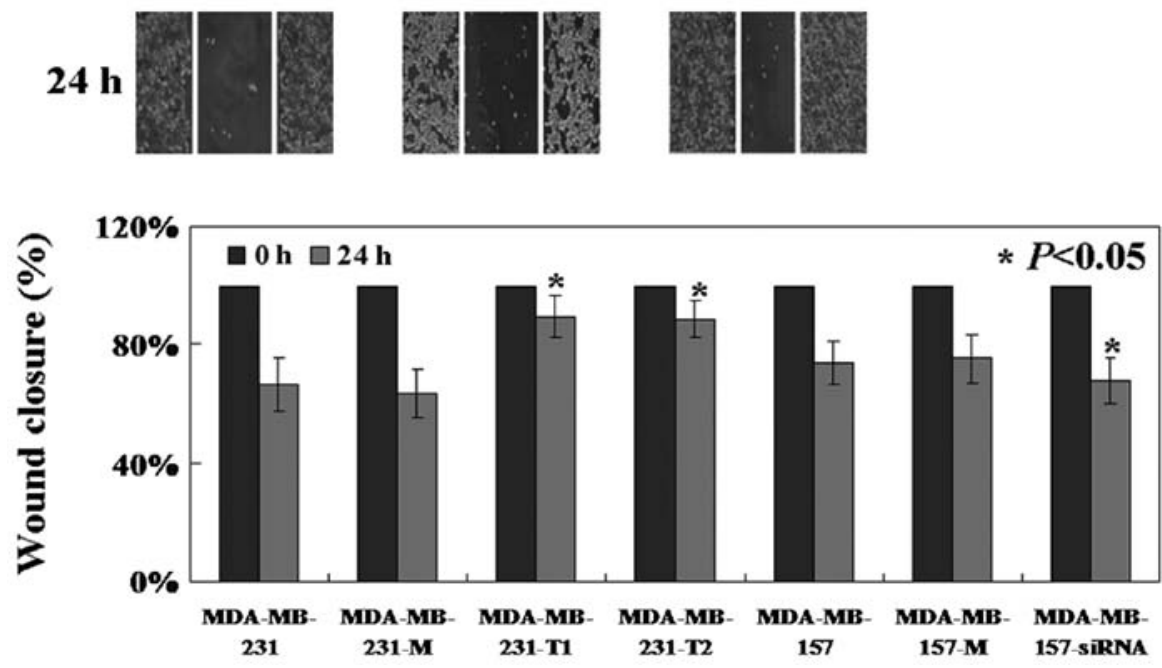

Figure 2. The antitumor activities of KISS1 in vitro. (A) The proportion of apoptotic cells (early apoptosis) was determined by double-staining with Annexin V/ FITC and PI. (B) Wound closure was quantified in the regions flanked by dotted lines. 


\section{C}
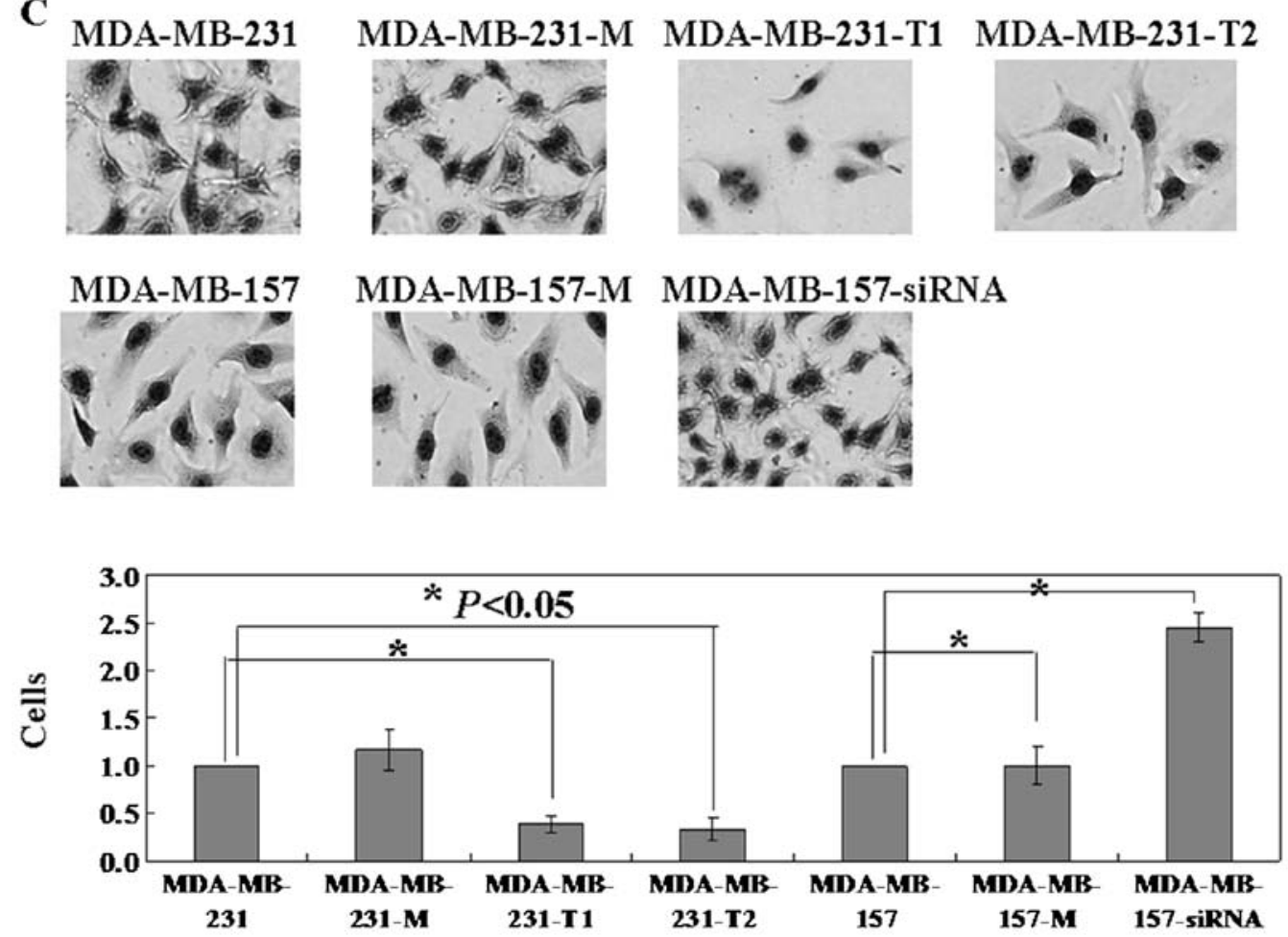

Figure 2. Continued. (C) The mobility of cells was determined by using transwell assay. All results were statistically significant $(\mathrm{P}<0.05)$.

weaker lamellipodia formation in MDA-MB-231 cells, labeled with phalloidin immunostaining. Downregulation of KISS1 protein in MDA-MB-157 cells showed stronger lamellipodia formation (Fig. 1C).

The antitumor activity of KISS1 protein in breast cancer mouse model. As shown above, KISS1 mediates an inhibitory effect on breast cancer cells in vitro. The antitumor properties of KISS1 were further evaluated using breast cancer mouse models. As shown in the Fig. 3A, upper panel, from days 10 to 60 , compared to untreated MDA-MB-231 cells and mock treated ones, intact or truncated KISS1 treated cells had a significant lower tumor volume $(\mathrm{P}<0.05)$. Correspondingly, the weights of intact or truncated KISS1 treated tumors also were lower than that of untreated and mock treated tumors $(\mathrm{P}<0.05$; Fig. 3A lower panel). In addition, the survival rate of mice with tumors treated with intact or truncated KISS1 was significantly improved. By the end of the experiment, 16/20 of mice in the intact or truncated KISS1 group were still alive, while all of the mice in the untreated and mock groups had died (Fig. 3B). Conversely, results using KISS1-knockdown MDA-MB-157 mouse models were comparable to results using the KISS1-upregulation MDA-MB-231 mouse models ( $\mathrm{P}<0.05$; Fig. 3).

Correlation of metastin serum levels or mouse model prognosis and CTCs. A statistically significant difference was observed between circulating metastin levels in each group $(\mathrm{P}<0.05$; Fig. 3C). Intact KISS1 treated group showed a higher serum metastin level than untreated group and mock treated group. However, truncated KISS1 treated group showed no changes compared with untreated group ( $\mathrm{P}>0.05$; Fig. 3C). CTC detection remains a big technical challenge despite the continuing development of many new exciting technologies (17). In this study, we isolated and enumerated the circulating tumor cells in mouse models by using the CellSearch system. KISS1-positive models showed less CTCs in peripheral blood than KISS1-negative ones $(\mathrm{P}<0.05$; Fig. 3D). The plasma level of metastin and the number of CTCs were significantly positively correlated $(r=-0.981, \mathrm{P}<0.001$; Fig. $3 \mathrm{E})$. To further investigate the relationship between CTC number and prognosis we defined 3 risk groups, a low (CTC <10), a medium (CTC 10-30) and a high-risk group (CTC >30). A significantly different survival rate between the low and the medium risk as well as between the medium and the high-risk groups in the Cox model was confirmed $(\mathrm{P}<0.05$; Fig. $3 \mathrm{~F})$.

KISS1 fragments suppress the MEK/ERK signaling pathway. To identify the mechanism of apoptosis induced by KISS1, immunohistochemical staining assays were performed to detect changes in possible signaling pathway proteins. While total levels of MEK and ERK showed no changes, the levels of phospho-MEK and phospho-ERK were observed to be significantly lower in KISS-positive cells versus negative ones (Fig. 4). Interesting, both intact and truncated KISS1 could inhibit the MEK/ERK signaling pathway in breast cancer cells. Activation of phospho-MEK and phospho-ERK were detected, while total levels of Ras, Raf, MEK and ERK were unchanged after MDA-MB-157 models treated with KISS siRNA (Fig. 4). In combination, these results suggest that KISS1 induced apoptosis in breast cancer cells by suppressing the MEK/ERK signaling pathway. 

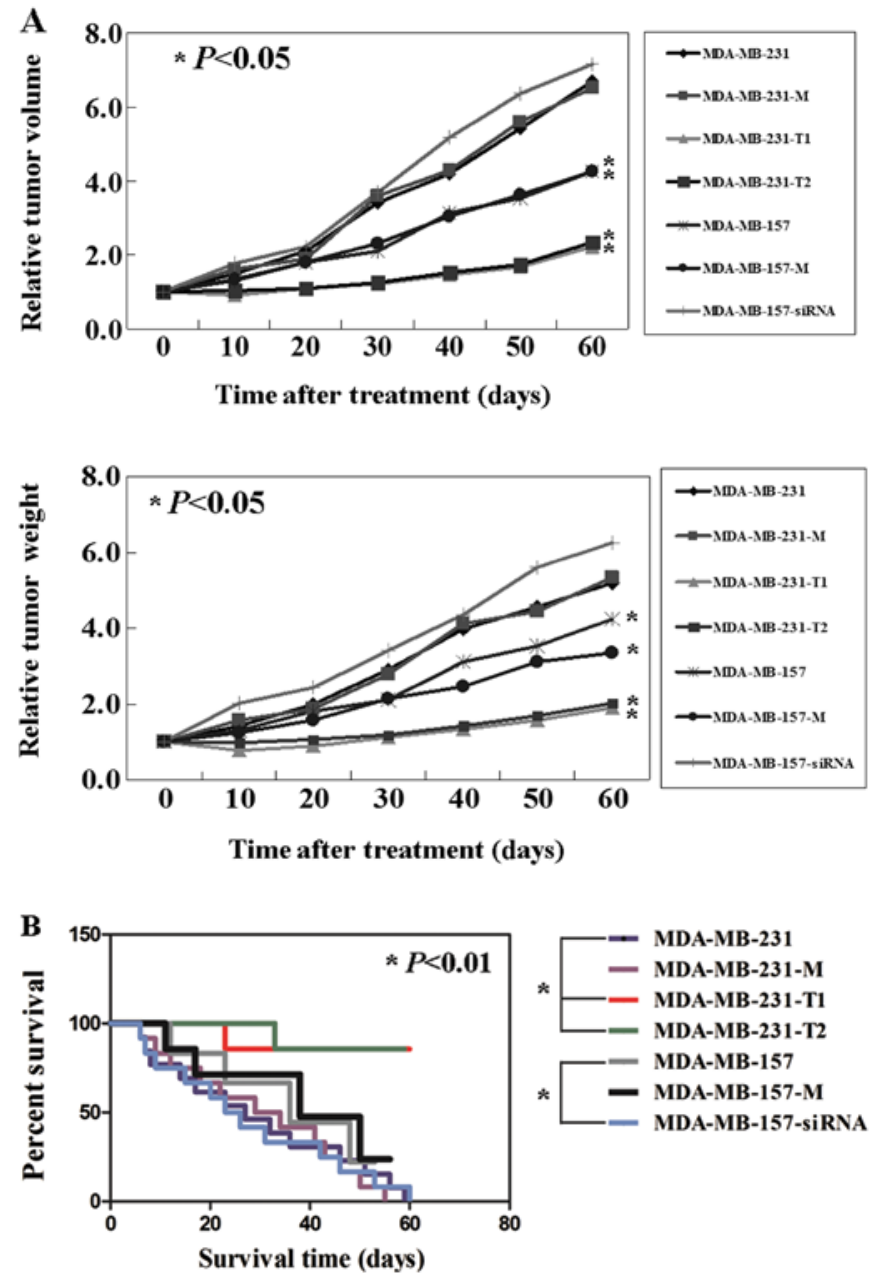

\section{Discussion}

Downregulation of KISS1 expression is able to increase tumor progression and poor prognosis in many cancers, such as pancreatic cancer, breast cancer, bladder cancer, brain cancer, epithelial ovarian cancer and gastric cancer (18-23). A recent study showed that KISS1 expression is decreased in human breast cancer, particularly in patients with aggressive tumors and with mortality (24). As noted in the introduction, kisspeptin is a product of the KISS1 gene which encodes a 145-amino acid precursor. Posttranslational modifications of this peptide result in a C-terminally amidated 54-amino acid peptide and several shorter fragments (e.g. kisspeptin-10, kisspeptin-13, kisspeptin-14) (6). However, the biological activities of these fragments were not very clear. In this study, we aimed to explicate the distinct roles of KISS1 different fragments in breast cancer cell. We studied two breast cancer cell lines, MDA-MB-231 and MDA-MB-157. Consistent with Teng et al (25), we confirmed MDA-MB-231 cells showed relatively low KISS1 expression levels, whereas the MDA-MB-157 expressed relatively stronger KISS1 protein levels. We found that KISS1 was aberrantly silenced by $\mathrm{CpG}$ island promoter hypermethylation. In bladder tumors (10) and colorectal tumors (11), the loss of KISS1 expression was attributed to epigenetic silencing by hypermethylation. Then we upregulated KISS1 protein expression in MDA-MB-231 cells by eukaryotic transfection and downregulated KISS1 protein expression in MDA-MB-157 cells by RNA interference. MDA-MB-231 cells after treatment showed a higher apoptotic ratio and a lower

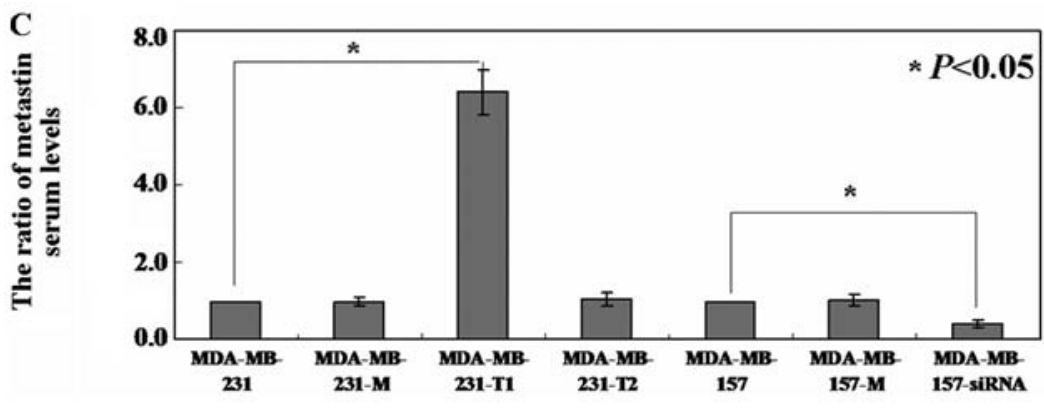

D

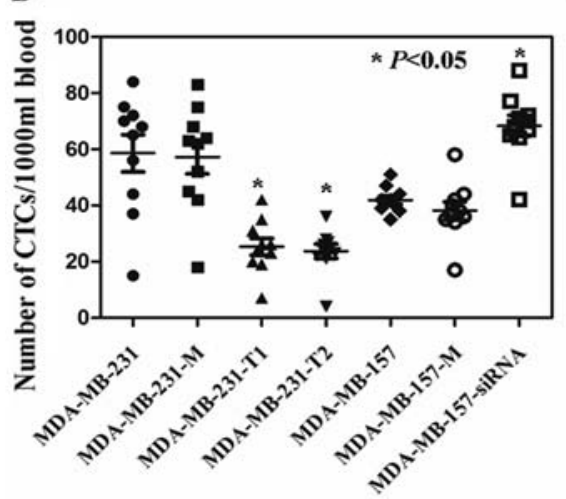

E

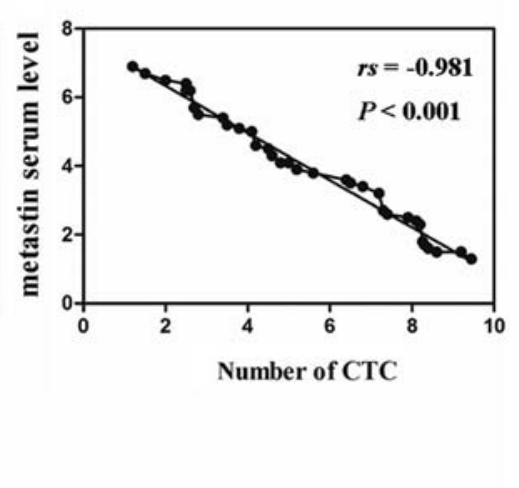

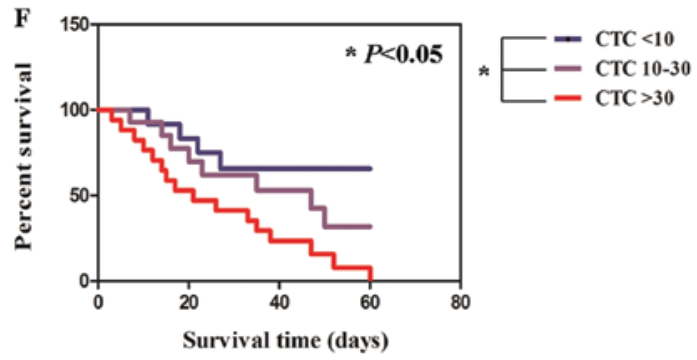

Figure 3. KISS1 suppressed tumor growth in xenograft mouse models. (A) Tumor volume and tumor weights of each group described in Materials and methods that were sacrificed 20 days after the cells were injected. (B) Kaplan-Meier survival curves of the groups described in Materials and methods. (C) Serum metastin levels from xenograft mouse models were measured by using ELISA. (D) Circulating tumor cells (CTCs) in serum of each group were isolated by using the CellSearch system. (E) Negative correlation between serum levels of metastin and CTC in xenograft mouse models. (F) Kaplan-Meier plot estimating overall survival for 3 risk groups ( $<10$ CTCs, $10-30$ CTCs, $>30$ CTCs). All results were statistically significant $(\mathrm{P}<0.05)$. 


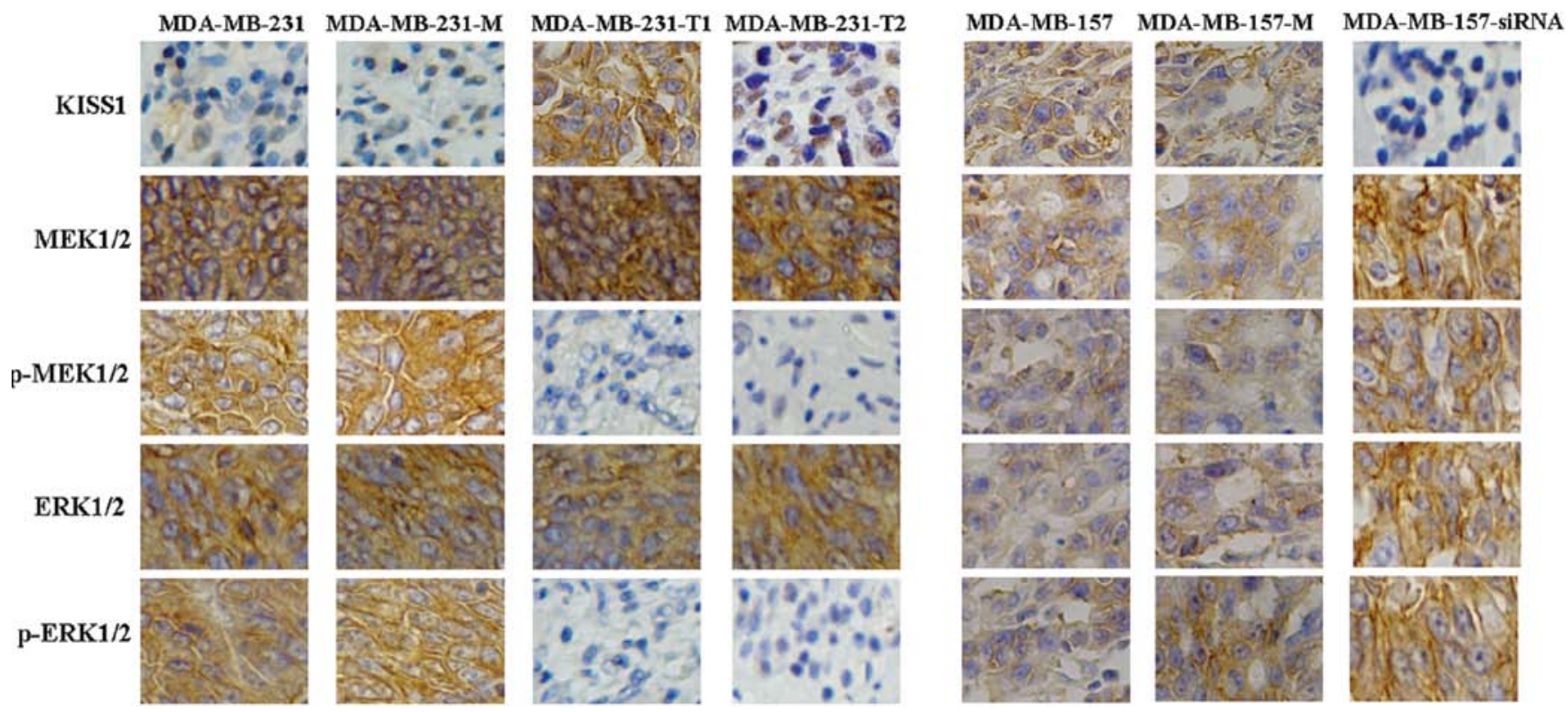

Figure 4. Immunohistochemical staining of the MEK/ERK signaling pathway. Levels of phosphorylated forms of MEK 1/2 and ERK 1/2 were almost disappeared in KISS positive cell lysates. However, the levels of total MEK 1/2 and ERK 1/2 remain unchanged. Consistent the results of immunofluorescence, intact KISS1 proteins were located in the cytoplasm and truncated KISS1 fragments were observed to localize in the nucleus. The nuclei were counterstained with hematoxylin.

mobility than untreated ones. Previous studies showed that an inverse relationship between of KISS1 and MMP-9 expressions $(26,27)$. We also confirmed that upregulation of KISS1 expression leads to loss of MMP-9 expression in breast cancer cell lines.

The main purpose of this study was to identify the roles of KISS1 protein distinct domains. Especially, nuclear export signal of KISS1 protein has not been formally identified. Interesting, we found KISS1 protein without residues 1-19 was unable to shift from nucleus to cytoplasm entirely. The results indicated that the nuclear export signal of KISS1 may be included in residues 1-19. However, KISS1 protein without residues 1-19 also showed antitumor activity. Both intact KISS1 protein and KISS1 $\Delta 20-121$ induced apoptosis, inhibited mobility and caused weaker lamellipodia formation. In vivo, tumor volumes and tumor weights were found to be reduced in xenografts established from treated cells versus untreated cells. Correspondingly, survival times were also longer for treated cell xenograft models. Previous studies have shown that patients with negative expression of KISS1 protein were correlated with poor disease-specific survival by using Kaplan-Meier curve survival analysis $(10,11)$. Metastasis is a multi-stage process that selects for circulating tumor cells (CTCs) that can infiltrate, survive in and colonize distant organs (28). Several studies have suggested that the presence of CTCs that have survived therapy might reflect a failure of systemic therapy $(29,30)$. Consistent with previous studies, we also found a negative correlation between the plasma level of metastin and the number of CTCs in xenograft models.

The mechanism of KISS1 suppression remains unclear. KISS1 protein could induce $\mathrm{Ca}^{2+}$ in receptor-transfected $\mathrm{CHO}$ cells, as well as phosphorylation of ERK1/2 and weak phosphorylation of p38/MAPK (31). Yan et al (32) found that metastin inhibits motility by repressing the transcription of
MMP-9 via the induction of cytosolic $\mathrm{I} \kappa \mathrm{B} \alpha$. Another study showed that metastin induces excessive formation of focal adhesions and stress fibers in hOT7T175-transfected B16/BL6 and induces the phosphorylation of FAK and paxillin (33). A further important finding of this study was that KISS1 induced apoptosis in breast cancer cells by suppressing the MEK/ERK signaling pathway. The Ras/Raf/MEK/MAPK pathway has been associated with a more aggressive disease or poor prognosis in variety of cancer systems $(34,35)$. Inactivation of the MEK/ERK pathway has been observed to increase cell death in studies of commonly used chemotherapy agents in vitro during the past few years $(36,37)$.

Taken together, both intact KISS1 protein and KISS1$\Delta 20$-121 were observed to mediate similar antitumor effects in breast cancer cell line and these involved suppression of the Ras/Raf/MEK/ERK signaling pathway. Moreover, following the inhibition of this signaling pathway, breast cancer cell lines showed higher apoptotic ratio, lower mobility and weaker lamellipodia formation in vitro. In vivo, tumor volumes and tumor weights were found to be reduced after KISS1 treatment. Additionally a negative correlation was confirmed between the plasma level of metastin and the number of CTCs in xenograft models. These results provide valuable insight into potential novel treatments for breast cancer.

\section{Acknowledgements}

We are indebted to Liu Ning for his helpful criticism of the manuscript and excellent technical assistance.

\section{References}

1. Salomon JA, Vos T, Hogan DR, et al: Common values in assessing health outcomes from disease and injury: disability weights measurement study for the Global Burden of Disease Study 2010. Lancet 380: 2129-2143, 2012. 
2. Jemal A, Bray F, Center MM, et al: Global cancer statistics. CA Cancer J Clin 61: 69-90, 2011.

3. Kushi LH, Byers T, Doyle C, et al: American Cancer Society Guidelines on Nutrition and Physical Activity for cancer prevention: reducing the risk of cancer with healthy food choices and physical activity. CA Cancer J Clin 56: 254-281, quiz 313-314, 2006.

4. Lee JH, Miele ME, Hicks D, et al: KiSS-1, a novel human malignant melanoma metastasis-suppressor gene. J Natl Cancer Inst 88: 1731-1737, 1996.

5. Makri A, Pissimissis N, Lembessis P, et al: The kisspeptin (KISS-1)/GPR54 system in cancer biology. Cancer Treat Rev 34 682-692, 2008

6. Ohtaki T, Shintani Y, Honda S, et al: Metastasis suppressor gene KiSS-1 encodes peptide ligand of a G-protein-coupled receptor. Nature 411: 613-617, 2001.

7. Muir AI, Chamberlain L, Elshourbagy NA, et al: AXOR12, a novel human $\mathrm{G}$ protein coupled receptor, activated by the peptide KiSS-1. J Biol Chem 276: 28969-28975, 2001.

8. Kotani M, Detheux M, Vandenbogaerde A, et al: The metastasis suppressor gene KiSS-1 encodes kisspeptins, the natural ligands of the orphan G protein-coupled receptor GPR54. J Biol Chem 276: 34631-34636, 2001.

9. Nash KT and Welch DR: The KISS1 metastasis suppressor: mechanistic insights and clinical utility. Front Biosci 11: 647-659, 2006.

10. Cebrian V, Fierro M, Orenes-Piñero E, et al: KISS1 methylation and expression as tumor stratification biomarkers and clinical outcome prognosticators for bladder cancer patients. Am J Pathol 179: 540-546, 2011.

11. Moya P, Esteban S, Fernandez-Suarez A, et al: KiSS-1 methylation and protein expression patterns contribute to diagnostic and prognostic assessments in tissue specimens for colorectal cancer. Tumour Biol 34: 471-479, 2013.

12. Cristofanilli M, Hayes DF, Budd GT, et al: Circulating tumor cells: a novel prognostic factor for newly diagnosed metastatic breast cancer. J Clin Oncol 23: 1420-1430, 2005.

13. Cristofanilli M, Budd GT, Ellis MJ, et al: Circulating tumor cells, disease progression, and survival in metastatic breast cancer. N Engl J Med 351: 781-791, 2004.

14. Osta WA, Chen Y, Mikhitarian K, et al: EpCAM is overexpressed in breast cancer and is a potential target for breast cancer gene therapy. Cancer Res 64: 5818-5824, 2004.

15. Gusterson BA, Ross DT, Heath VJ, et al: Basal cytokeratins and their relationship to the cellular origin and functional classification of breast cancer. Breast Cancer Res 7: 143-148, 2005.

16. Allard WJ, Matera J, Miller MC, et al: Tumor cells circulate in the peripheral blood of all major carcinomas but not in healthy subjects or patients with nonmalignant diseases. Clin Cancer Res 10: 6897-6904, 2004.

17. Pantel K, Brakenhoff RH and Brandt B: Detection, clinical relevance and specific biological properties of disseminating tumor cells. Nat Rev Cancer 8: 329-340, 2008.

18. Masui T, Doi R, Mori T, et al: Metastin and its variant forms suppress migration of pancreatic cancer cells. Biochem Biophys Res Commun 315: 85-92, 2004.

19. Martin TA, Watkins G and Jiang WG: KiSS-1 expression in human breast cancer. Clin Exp Metastasis 22: 503-511, 2005.

20. Nicolle G, Comperat E, Nicolaïew N, et al: Metastin (KISS-1) and metastin-coupled receptor (GPR54) expression in transitional cell carcinoma of the bladder. Ann Oncol 18: 605-606, 2007.
21. Zohrabian VM, Nandu H, Gulati N, et al: Gene expression profiling of metastatic brain cancer. Oncol Rep 18: 321-328, 2007.

22. Hata K, Dhar DK, Watanabe Y, et al: Expression of metastin and a G-protein-coupled receptor (AXOR12) in epithelial ovarian cancer. Eur J Cancer 43: 1452-1459, 2007.

23. Yamashita S, Tsujino Y, Moriguchi K, et al: Chemical genomic screening for methylation-silenced genes in gastric cancer cell lines using 5-aza-2'-deoxycytidine treatment and oligonucleotide microarray. Cancer Sci 97: 64-71, 2006.

24. Mooez S, Malik FA, Kayani MA, et al: Expressional alterations and transcript isoforms of metastasis suppressor genes (KAI1 and KiSS1) in breast cancer patients. Asian Pac J Cancer Prev 12: 2785-2791, 2011

25. Teng Y, Liu M and Cowell JK: Functional interrelationship between the WASF3 and KISS1 metastasis-associated genes in breast cancer cells. Int J Cancer 129: 2825-2835, 2011.

26. Dhar DK, Naora H, Kubota H, et al: Downregulation of KiSS-1 expression is responsible for tumor invasion and worse prognosis in gastric carcinoma. Int J Cancer 111: 868-872, 2004

27. Zheng S, Chang Y, Hodges KB, et al: Expression of KISS1 and MMP-9 in non-small cell lung cancer and their relations to metastasis and survival. Anticancer Res 30: 713-718, 2010.

28. Pantel K and Brakenhoff RH: Dissecting the metastatic cascade. Nat Rev Cancer 4: 448-456, 2004.

29. Müller V, Stahmann N, Riethdorf S, et al: Circulating tumor cells in breast cancer: correlation to bone marrow micrometastases, heterogeneous response to systemic therapy and low proliferative activity. Clin Cancer Res 11: 3678-3685, 2005.

30. Slade MJ, Payne R, Riethdorf S, et al: Comparison of bone marrow, disseminated tumour cells and bloodcirculating tumour cells in breast cancer patients after primary treatment. Br J Cancer 100: 160-166, 2009.

31. Lee YR, Tsunekawa K, Moon MJ, et al: Molecular evolution of multiple forms of kisspeptins and GPR54 receptors in vertebrates. Endocrinology 150: 2837-2846, 2009.

32. Yan C, Wang H and Boyd DD: KISS-1 represses 92-kDa type IV collagenase expression by down-regulating NF-kappa B binding to the promoter as a consequence of Ikappa Balpha-induced block of p65/p50 nuclear translocation. J Biol Chem 276: 1164-1172, 2001.

33. Mandache E, Gherghiceanu M, Serafinceanu C, et al: Myofibroblast involvement in tubular basement membrane remodeling in type II diabetic nephropathy. Rom J Morphol Embryol 52: 75-79, 2011.

34. Reungwetwattana T, Weroha SJ and Molina JR: Oncogenic pathways, molecularly targeted therapies, and highlighted clinical trials in non-small-cell lung cancer (NSCLC). Clin Lung Cancer 13: 252-266, 2012.

35. Salomon DS, Brandt R and Ciardiello F: Epidermal growth factor-related peptides and their receptors in human malignancies. Crit Rev Oncol Hematol 19: 183-232, 1995.

36. Woessmann W, Chen X and Borkhardt A: Ras-mediated activation of ERK by cisplatin induces cell death independently of p53 in osteosarcoma and neuroblastoma cell lines. Cancer Chemother Pharmacol 50: 397-404, 2002.

37. Bacus SS, Gudkov AV, Lowe M, et al: Taxol-induced apoptosis depends on MAP kinase pathways (ERK and p38) and is independent of p53. Oncogene 20: 147-155, 2001. 